\title{
FUNDAMENTAL PRIORITIES IN POLLICISATION
}

\author{
W. F. White, Uddingston, Scotland \\ From the Orthopaedic Department, Law Hospital, Carluke
}

It has been said that, at best, pollicisation gives a hand that is cosmetically distasteful and functionally disappointing (Brooks 1966). This criticism is valid and even if it seems unduly harsh to some, it provides a timely and necessary reminder that a transposed finger never matches a thumb in performance or appearance. In this light it seems rational therefore to simplify the operation as much as possible and to be wary, especially in the congenital case, lest technical complexity designed to attain the versatility of the normal thumb leads in the end to poorer function.

\section{MATERIAL}

A small personal series of fourteen pollicisations has been reviewed. Five of these were carried out for traumatic amputation and nine for congenital absence. The study was made not primarily with a view to assessing results, but rather to try to set down the fundamental priorities in the operation, to point out the difficulties encountered, and to suggest how these might be overcome in as simple and straightforward a way as possible (White 1969).

\section{FUNDAMENTAL PRIORITIES}

Four basic prerequisites emerge as essential to the creation of a substitute for the thumb if it is to add any worthwhile improvement in function. First, and of course most important, survival of the transposed finger itself, but also, even if of less value, survival of skin flaps. Secondly, perfect sensibility, without which the finger cannot be put to any real use. Thirdly, good position, which is critical if the "thumb" is to stand as an opponent for the fingers. Fourthly, stability and strength, which are indispensable to resist the combined power of the other fingers (Littler 1953). Many other factors play their part, but in this series any pollicised finger that did not satisfy these fundamental requirements did not help significantly the use of the hand.

\section{SURVIVAL}

Possible loss of the finger is the greatest worry to any surgeon. This was one reason why the index finger was used in all cases. It is adjacent and therefore its transfer is more simple and damage to vessels is less likely.

Elevation of the hand, without the use of an Esmarch bandage, before application of the pneumatic tourniquet has led to very much easier identification of vessels and nerves. So, too, has early exposure from the palmar surface of the neurovascular bundles to the contiguous sides of the index and middle fingers (Murray 1946). The artery to the radial side of the middle finger was ligated as far distal to the bifurcation as possible, while the nerve was separated proximally if necessary (Fig. 1). Only then was it possible to move the index finger away from the middle finger.

Venous drainage appeared adequate so long as the hand was kept elevated after operation. The only finger that gave anxiety had been left dependent for a short time but it slowly recovered with elevation. Preservation of a dorsal vein, on which much emphasis has been laid, appeared to make no difference.

All the fingers survived but not all the flaps (Fig. 2). There was sometimes trouble with the web flap which did not swing easily on its palmar base because of the adherent quality of 
palmar skin. Indeed it was felt that one of the main advantages of recession-shortening the finger at its base-was to make skin closure easier (McGregor 1966).

A different approach was therefore used in the cases of congenital absence (Fig. 3). A racquet incision was made round the base of the index finger with the handle carried proximally along the proposed line of the new web on the palmar surface. This incision was broken up

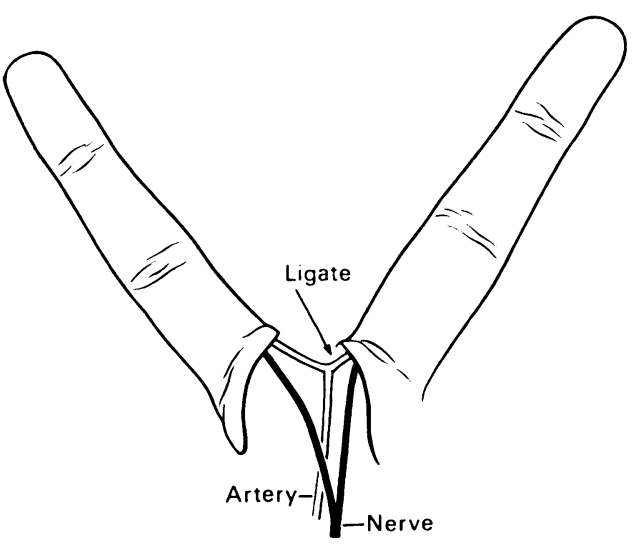

Fig. 1

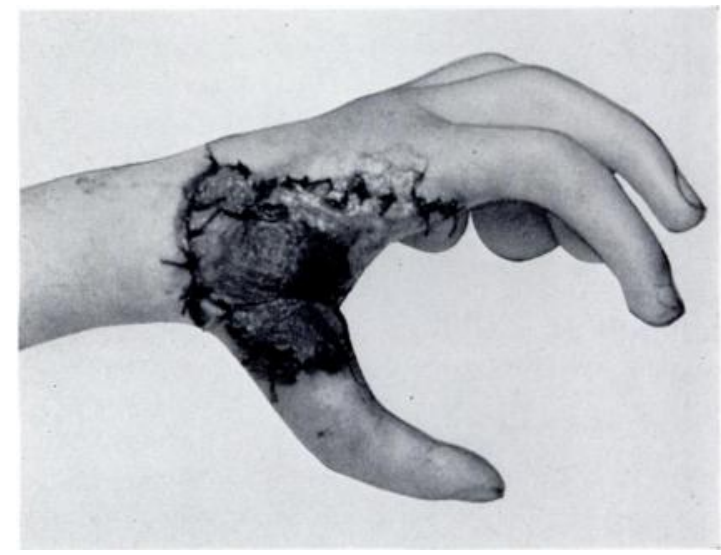

Fig. 2

Figure 1-Palmar exposure of the vessels and nerves to contiguous sides of the index and middle fingers. The fingers will not separate farther unless the artery to the radial side of the index finger is ligated and divided. The site is marked. Figure 2-Necrosis of a large part of the web flap.

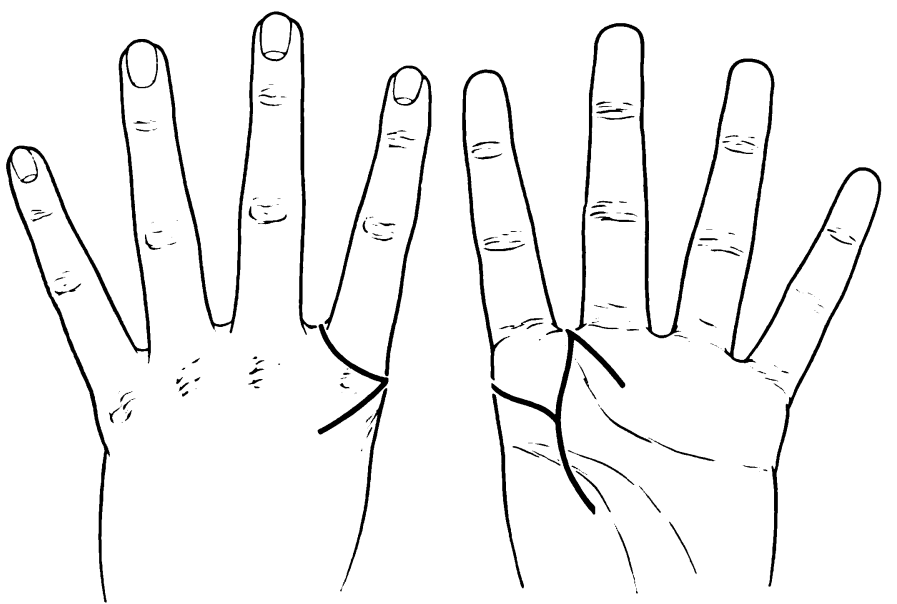

Fig. 3

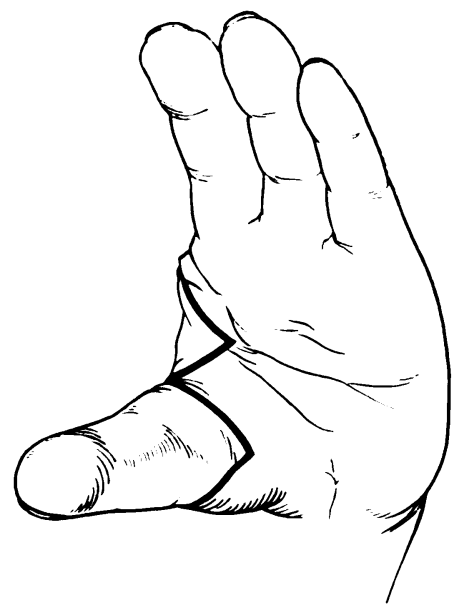

Fig. 4

Figure 3-Racquet type of incision showing Z-plasty. The incision is marked only on the presenting aspect of the hand. Figure 4-Closure of the Z-plasty.

by a Z-plasty. The first limb of this Z-the more important one-was on the palmar surface and was a great help in exposing the vessels and nerves. The second limb of the $Z$ was on the dorsal surface and aided exposure of the extensor tendons and index metacarpal. This incision made exposure and closure much easier (Fig. 4).

\section{SENSIBILITY}

This second priority - sensibility - is adequately safeguarded by the same measures which have been suggested to ensure survival of the finger.

vol. 52 B, No. 3, AUGUST 1970 


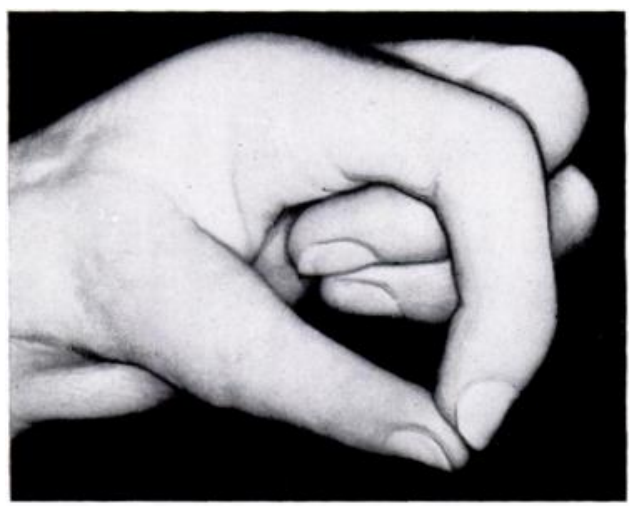

FIG. 5

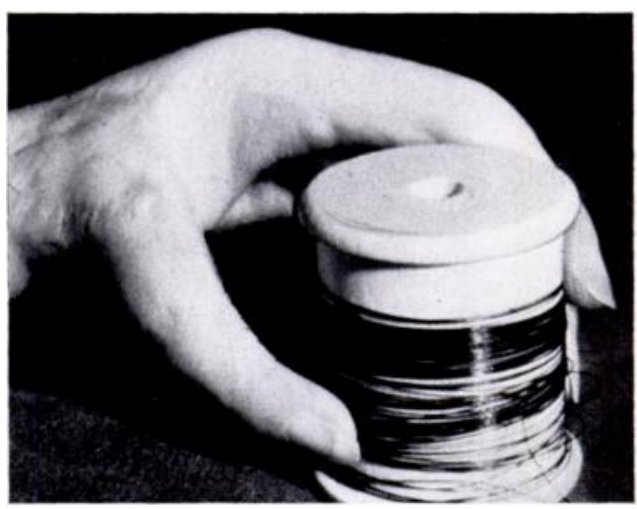

FIG. 6

Pulp to pulp opposition and wide grip.

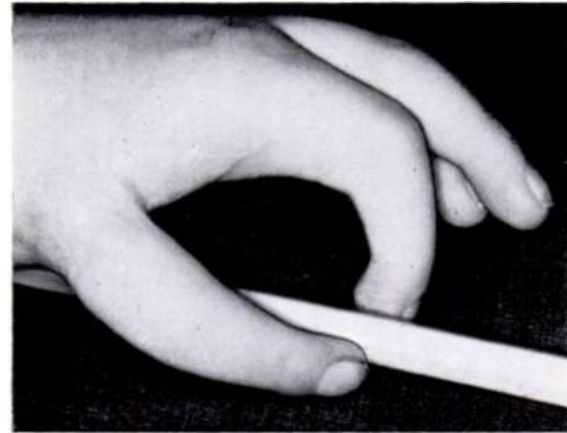

Fig. 7

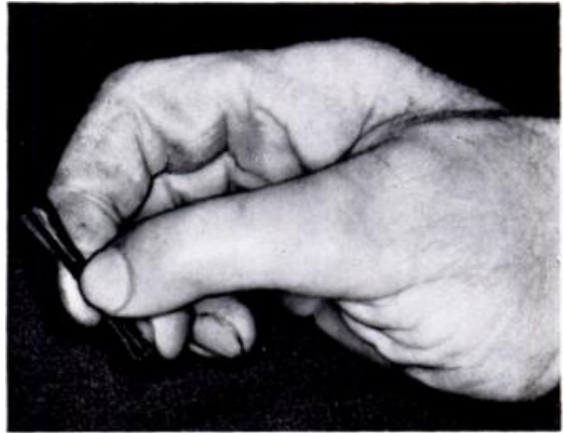

FIG. 8

Figure 7-Incorrect rotation and projection. Figure 8-Good function but there should have been more rotation.

\section{POSITION}

The aim has been to get the pulp of the transposed finger to oppose the pulp of the middle finger just a little on its radial side and to have a wide grip too (Figs. 5 and 6). This should be possible in the congenital cases but was not always achieved. A pinch grip more to the side of the middle finger was not so satisfactory (Fig. 7). This has generally meant that the rotation of the finger has been wrong and that the projection in a palmar direction may have

TABLE I

Position of TRANSPOSED Digit

\begin{tabular}{|c|cr|}
\hline Type & \multicolumn{2}{c|}{ Degrees } \\
\hline Congenital & Rotation & +150 \\
\cline { 3 - 3 } & Palmar projection & 40 \\
\cline { 2 - 3 } Traumatic & Rotation & 130 \\
\hline
\end{tabular}

been wrong as well. In traumatic cases the result is influenced by what thenar muscles are left and also by other soft-tissue damage. Figure 8 shows a case in which the functional result was good, and indeed the man went back to work as a coachbuilder: nevertheless the degree of rotation is a little less than it should be. Figures 9 and 10 show a case in which the original injury was much more severe. The patient sustained partial amputations of both index and 


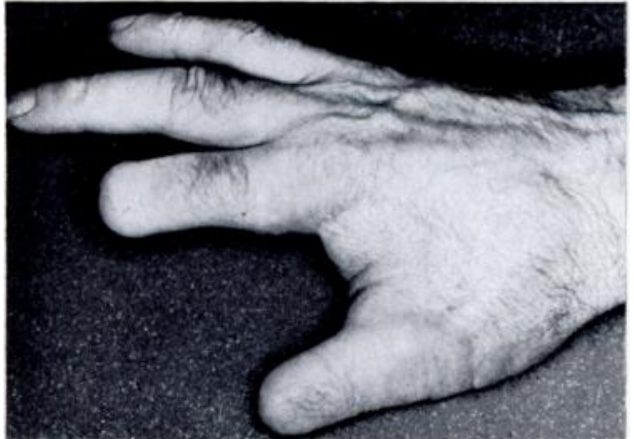

Fig. 9

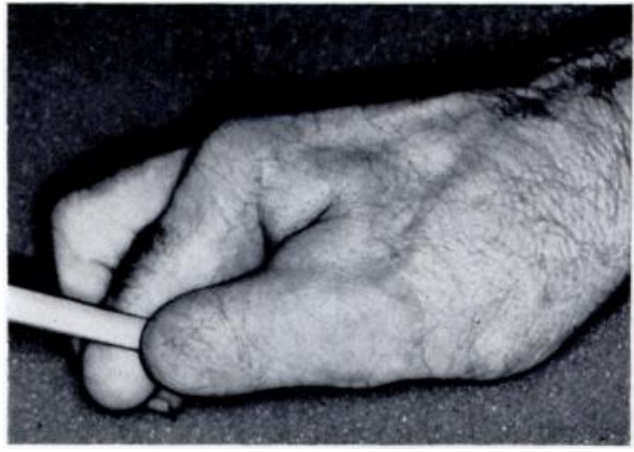

Fig. 10

A much more severe type of injury requiring web reconstruction.

middle fingers as well as a thumb amputation, and there was dense web scarring which necessitated reconstruction of the web by cross-arm flap. Although the functional result was gratifying and the patient had good use of the hand, the result naturally reflected the severity of the original damage.

In the series as a whole the best results were those in congenital cases in which the rotation

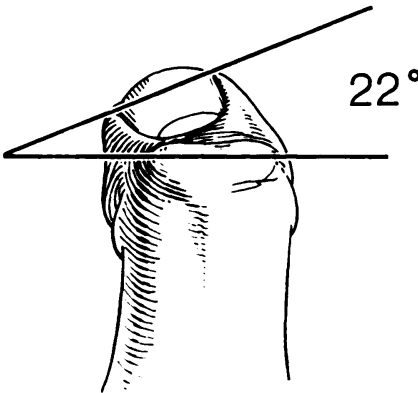

Fig. 11

Medial rotation of the distal segment of the right thumb-viewed from the dorsal aspect with the interphalangeal joint flexed. was 150 degrees or more and the projection in a palmar direction was about 40 degrees. In the traumatic cases the best rotation appeared to be about 130 degrees. These angles are much greater than those generally advised (Table I).

This amount of rotation, in the congenital cases, was used principally because of the belief that no transplant could emulate the thenar muscles and that flexion and extension might be the only worthwhile movements. In radial club hand even these are often poor.

This belief was sustained, if not engendered, by the realisation that there are a number of factors which enhance normal opposition but which are denied the pollicised finger. Firstly, it is never possible to reproduce the rotation of the proximal phalanx of the thumb brought about by the abductor pollicis brevis. Secondly, the distal segment of the thumb is medially rotated by an amount which varies with the degree of flexion-it may be up to 20 to 25 degrees in full flexion (Fig. 11), whereas the distal segment of the index may have a slight rotation in the opposite direction. In contradistinction to this it is freely admitted that in some cases of congenital absence of the thumb, the index finger, striving to behave like a thumb, does undergo an appreciable rotation towards the middle finger.

Perhaps the most significant point of all, which is much less easily defined, has reference again only to the congenital cases. The perfect opposition with stability always achieved by the normal thumb depends not so much on muscles as on the special shape of the carpo-metacarpal joint and on its ligaments. The thumb is guided unerringly to the correct rotation (Napier 1966). A pollicised finger in the congenital case has no such aid and the value of transplants is thereby lessened.

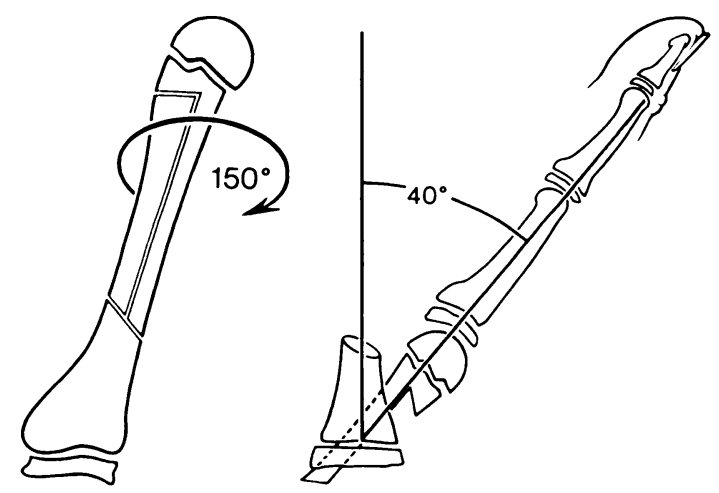

Fig. 12

Method used for proximal junction in a congenital case. 


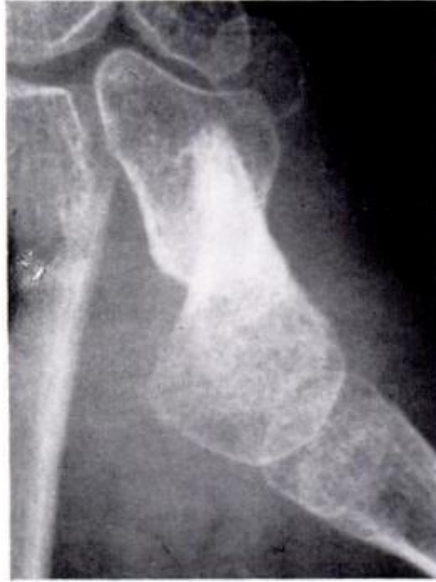

FIG. 13

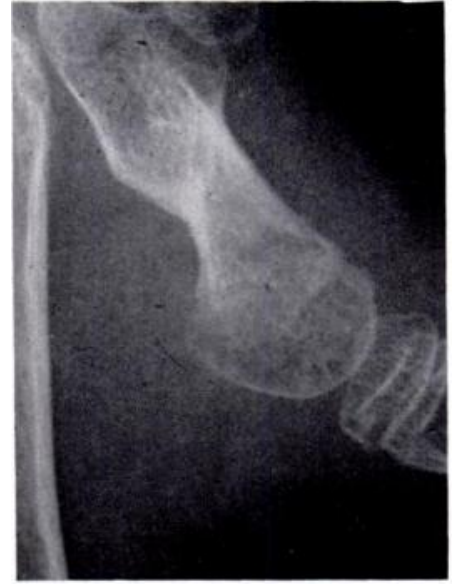

Fig. 14

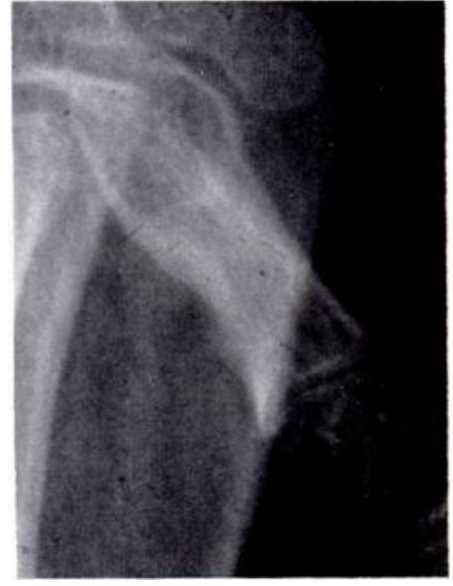

Fig. 15

Radiographs of the bony junction two, four and nineteen months after operation.

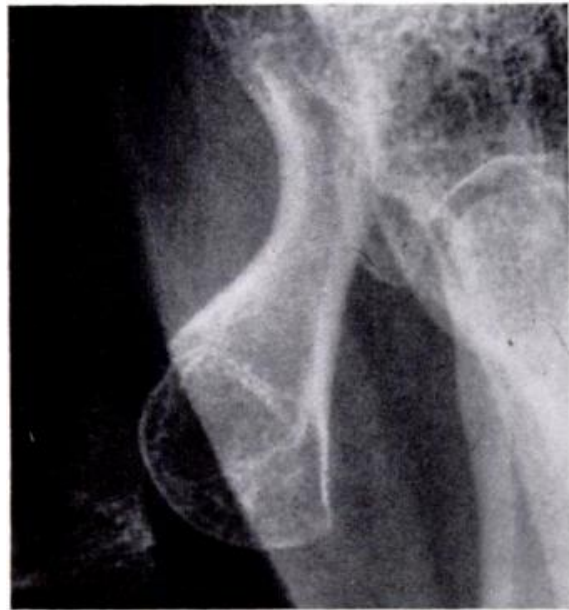

FiG. 16

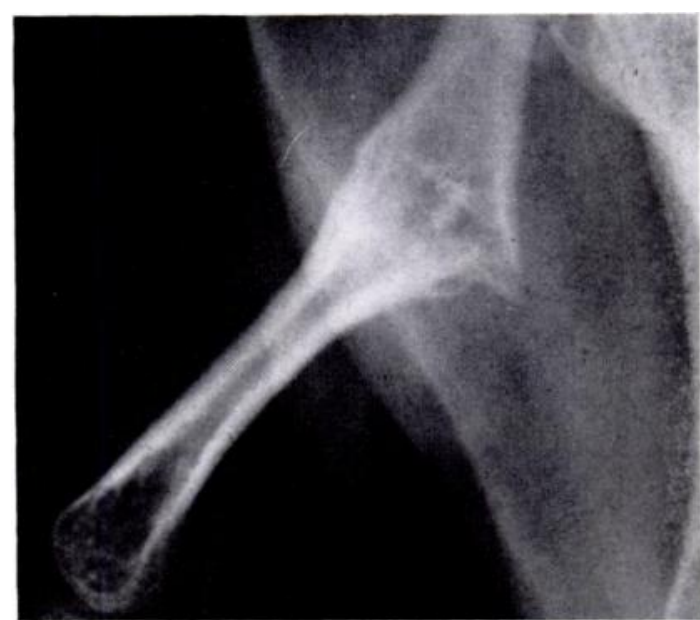

FIG. 17

Hyperextension at the old metacarpo-phalangeal joint which required arthrodesis.

\section{STABILITY AND STRENGTH}

The proximal junction, which must be stable, is closely linked with the problem of position. In the traumatic cases the usual method has been to use part of the shaft of the index metacarpal as an intramedullary graft. It did not prove easy to get a really tight fit with the rotation correct.

The junction is even more vital in the congenital cases. In this group it was not found possible to secure the index metacarpal into the carpus as has been suggested, to obtain a more normal "take off" and a better metacarpal angle (Edgerton, Snyder and Webb 1965).

The index metacarpal was split longitudinally by a saw cut extending from the neck towards the base for a distance of more than an inch. It was not quite centrally placed (Fig. 12). The thicker part was divided at neck level and the whole thickness at the proximal end. This produced a kind of spike graft about an inch long. It was put into a drill hole near the base of the metacarpal which might extend into the carpus. Everything depends, firstly, on this drill hole, which must start on the volar surface with the drill at an angle of about 40 degrees to the line of the metacarpal, and, secondly, on the rotation of the index finger, which must be checked before the spike is fixed in the hole. It has to be a firm and secure fit (Figs. 13 to 15). 
Even when this proximal junction is solid, the new thumb may not stand up to firm pressure against the fingers in a strong pinch grip but may buckle at the old metacarpophalangeal joint, which is now taking the place of a carpo-metacarpal joint (Buck-Gramcko 1967). It may require fusion (Figs. 16 and 17).

\section{SUMMARY}

1. A small series of fourteen pollicisations has been studied.

2. The keyword, so far as the operation is concerned, has been simplicity.

3. It has been appreciated that a transposed finger can never become a thumb, and it may not be wise to strive too hard by means of transplants to emulate the perfection of the normal thumb, especially if this is done at the expense of one of the fundamental priorities.

4. Nevertheless, if sensibility, good position and proximal stability are achieved, pollicisation can provide a useful addition to hand function and a reasonably satisfactory appearance.

I would like to express my thanks to $\mathrm{Mr}$ Athol Parkes for his encouragement and for referring a number of these patients to me for treatment; to Mr R. G. Scott, Superintendent Radiographer at Law Hospital, for the photographic work: to Mr Gabriel Donald of the Department of Medical Illustration in the Western Infirmary, Glasgow; and to Mrs Priscilla Miles, for the drawings.

\section{REFERENCES}

Brooks, D. (1966): Reconstruction of the Injured Hand. In Rehabilitation of the Hand by C. B. Wynn Parry. Second edition, p. 341. London: Butterworths.

Buck-Gramcko, D. (1967): Difficulties in Technique of Pollicisation of the Index Finger in Aplasia of the Thumb. [Proceedings of] The British Club for Surgery of the Hand, Vienna, May 29-30, 1967, p. 24.

Edgerton, M. T., SNyder, G. B., and WebB, W. L. (1965): Surgical Treatment of Congenital Thumb Deformities (Including Psychological Impact of Correction). Journal of Bone and Joint Surgery, 47-A, 1453.

LitTler, J. W. (1953): The Neurovascular Pedicle Method of Digital Transposition for Reconstruction of the Thumb. Plastic and Reconstructive Surgery, 12, 303.

MCGregor, I. A. (1966): Reconstruction of the Thumb. In Modern Trends in Plastic Surgery, 2, edited by Thomas Gibson, p. 178. London: Butterworths.

MurRay, A. R. (1946): Reconstructive Surgery of the Hand with Special Reference to Digital Transplantation. British Journal of Surgery, 34, 131.

NAPIER, J. R. (1966): Functional Aspects of the Anatomy of the Hand. In Clinical Surgery, 7, edited by C. Rob and R. Smith. The Hand, p. 19. Edited by R. G. Pulvertaft. London: Butterworths.

White, W. F. (1969): Pollicisation for the Missing Thumb, Traumatic or Congenital. The Hand: Journal of the British Society for Surgery of the Hand, 1, 23. 